\title{
The objective case for conservation
}

\author{
Not before time, people are beginning to wrestle with the concept of biodiversity not simply from academic \\ inclination, but so as to forge a practical yardstick for conservation.
}

Two neologisms beginning with ' $b$ ' have crept into general use in the past year or so: 'buckyballs' and 'biodiversity'. What the former lacks in elegance, the latter lacks in definition. Which is odd, considering that 152 world leaders have recently signed their names to a pledge to protect biodiversity, whatever it may be. But that circumstance was enough to lift the hearts of participants at a meeting* in London last week (planned long before the Rio summit) designed to grapple with the task of defining biodiversity, how to measure it, and how then to conserve it. ('Biodiversicist' may well be among next year's crop of unwieldy neologisms.)

But measuring biodiversity means more than making a list of species. Species are in any case disappearing more quickly than they can be formally named, so that a comprehensive inventory would arguably be more useful to a recording angel than to conservationists working to save what are left. Formal lists are out - "quick and dirty" lists (in the words of Robert M. May of the University of Oxford) are in. But such lists must include details of habitat, range, conservation status and phylogenetic relationship as well as a taxonomic label.

Inventories with these features are already beginning to be compiled and used, in particular to identify biodiverse 'hotspots' - circumstances, topographical or otherwise, notable for their diversity. It matters little that some of this information may be missing, because cross-referencing of what information there is should be enough to inform provisional decisions about conservation priorities. Time, money and manpower are in any case brakes on what can be done.

Biodiversity, then, is an epiphenomenon, an expression of the interaction of a variety of biotic and abiotic factors. Redundancy in the system ensures that the conservation of biodiversity remains valid as an ideal, even when many (or most) natural habitats have disappeared.

Accordingly, biodiversity embraces far more than taxonomy, although access to biological collections and taxonomic expertise is still central. This is reflected by the diversity of approaches now used to measure biodiversity, which can be quantified by the span of phylogenetic relatedness (the more, the more diverse), using cladistic or cytogenetic language; by the patchiness of

* Systematics \& Conservation Evaluation, Natural History Museum, London, 17-19 June 1992.

NATURE · VOL $357 \cdot 25$ JUNE 1992 habitats and biogeographical ranges; by the abundances of key 'indicator' species; by the identification of particularly important ecological interactions; or combinations of some or all these approaches.

Inevitably, many of the yardsticks being canvassed are mathematical in character, not entirely in tune with the popular image of the conservationist as a person with a big heart and, if a man, with a chunky hand-knitted sweater and a beard. The immediate task is to ground emotion in objective measures. The goal, throughout, is the same: how to conserve the greatest amount of biodiversity in the smallest area, and so (presumably) at the lowest possible cost.

But conservation policies, however wellrooted in science, can work only if they are intelligible within the diverse cultural contexts of those required to follow and to enforce them. To parrot Marx, conservation policy is both idiosyncratic and eclectic. It may follow that the meaning of biodiversity itself is coloured by cultural referents. That a single word can encompass so much may be sufficient excuse for both its awkwardness and its vagueness.

But why conserve biodiversity at all? It is difficult to argue for conservation if biodiversity is represented only by a list of species, some (but not all) of which may be technically endangered. Of course, there may be moral or aesthetic grounds for the conservation of individual species, but even these may differ between cultures (witness the annual conflict, about to be resumed in the International Whaling Commission, between whaling nations and others). Similarly, when people from rich countries decry the loss of habitat elsewhere, they may find their arguments cut no ice with those to whom habitat loss may mean the key to short-term economic survival.

Although the loss of the African elephant or the blue whale would be sad, there is no particular reason to spare these species in particular if they are just two among many millions. But to argue that the blanket preservation of millions of species might safeguard the few that, at some unknown future date, might prove useful in agriculture or medicine sounds like special pleading. Such possibilities are by-products of some elements of biodiversity, and offer no good reasons for conserving biodiversity in totality.

Far more convincing arguments stem from the concept of biodiversity as the sum of the interactions between species, rather than a list of the species themselves. More than this, these arguments follow from this opera- tional concept quite naturally, and do not have to be tacked on as afterthoughts to justify the effort.

That biodiversity defines a working system rather than a list of parts means that the conservation of as many parts of it as possible allows the system as a whole to retain flexibility (which can be a hedge against change) as well as preserving diversity for future scientific study or commercial exploitation. Importantly, these changes embrace far more than natural variations in climate.

There are, of course, some who argue that conservation is futile, given that evolution and extinction are natural consequences of climate change, irrespective of human interference. But that argument overlooks the vested interest of human populations in keeping things very much as they are, which implies that conservation has a natural place in economic strategy. 'Conservation', after all, has the same etymological roots as 'conservative' (with a small 'c').

It follows from this that biodiversity plays a real part in the material well-being of people. How can this be? Few will be able to identify - let alone quantify - any coarsening in their quality of life as a result of the extinction of this species or that. But it is certainly the case that the physical circumstances that allow us to live and breathe on the Earth are in no small measure the products of biodiversity, in the sense that biodiversity is a system rather than a list. Biodiverse interactions over billions of years have ensured that we have an atmosphere to breathe, a soil to till and fresh water to drink (and that we ourselves are adapted to the use of these materials, not to others not in existence). The integrity of this system as a whole transcends the extinction of particular species, even if some species may be more important than others for the coherence of the overall fabric.

The pollution of the atmosphere, the denudation of soil and the diminution of supplies of fresh water in many parts of the world could be seen, in part, as consequences of the loss of biodiversity. As longtime environmental campaigner David Bellamy put it at the start of last week's meeting, the effects are already being felt. Economic hardship and civil strife may be consequences, in part, of the neglect of conservation as well as its continuing causes. Perhaps govern-ments' acknowledgement of that explains why the biodiversity treaty gathered so many signatures at Rio. The worry is that they may not have been fully aware of what the concept means.

Henry Gee 